\title{
Métodos de amostragem de solos em áreas sob plantio direto no Sudoeste Goiano
}

\author{
Nelson H. D. Acqua1, Gilson P. Silva², Vinicius M. Benites ${ }^{3}$, \\ Renato L. de Assis ${ }^{4}$ \& Gustavo A. Simon ${ }^{5}$
}

\begin{abstract}
RESUMO
A amostragem de solo é a etapa mais importante do programa de avaliação da sua fertilidade; ressalta-se que a recomendação de corretivos e de fertilizantes é definida a partir da interpretação dos resultados de análises químicas realizadas na amostra. Com o objetivo de avaliar esses resultados em função do método de amostragem amostras de solo foram retiradas em 12 áreas distintas cultivadas há 2, 5 e 10 anos sob o sistema plantio direto. Utilizaram-se o trado holandês e a furadeira elétrica retirando-se as amostras de solo em 3 posições e 2 profundidades e se avaliando a fertilidade do solo em função do tempo de adoção do sistema plantio direto. Constatou-se incremento do teor de $\mathrm{P}$ nas áreas cultivadas há 10 anos sob plantio direto, em relação às demais. Os valores de $\mathrm{Ca}, \mathrm{Mg}, \mathrm{P}, \mathrm{K}, \mathrm{pH}, \mathrm{CTC}$ e $\mathrm{V}$ apresentaram gradiente de concentração em função do não revolvimento do solo, aplicações superficiais de calcário e adubações na linha de plantio. Os equipamentos de amostragem influíram nos resultados das análises de solo sendo que todos os macronutrientes, com exceção do S, e todos os micronutrientes, apresentaram valores elevados quando amostrados com a furadeira elétrica.
\end{abstract}

Palavras-chave: análise de solo, fertilidade do solo, gradiente, equipamentos

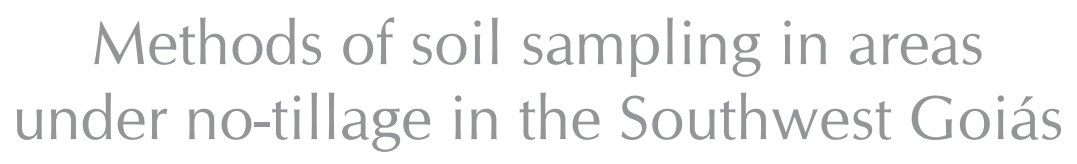

\begin{abstract}
Soil sampling is the first and fundamental step in an evaluation program of soil fertility, and the recommendation of lime and fertilizer is defined through the interpretation of the results of chemical analysis of the sample. With the objective of evaluating the results of the chemical analysis of the soil as a function of the sampling method, soil samples were collected from 12 different areas that have been under no-tillage system for 2, 5 and 10 years. The Ducth auger hole and the electric drill were used to collect the soil samples at 3 different positions and 2 depths, evaluating the soil fertility as a function of time of the direct planing system. There was an increment of $P$ in areas with 10 years under no-tillage system in comparison with the others areas. The values of $\mathrm{Ca}, \mathrm{Mg}, \mathrm{P}, \mathrm{K}, \mathrm{pH}, \mathrm{CTC}$ and $\mathrm{V}$ showed concentration gradient as a function of time of no tillage, superficial application of lime and fertilization in the planting rows. The sampling equipment influenced the results of soil analysis, and all macronutrients except $S$ and all micronutrients, showed higher values when soil was sampled with the electric drill.
\end{abstract}

Key words: soil analysis, soil fertility, gradient, equipments

\footnotetext{
${ }^{1}$ Apagri Consultoria Agronômica Ltda - R. Dona Eugênia 957, CEP 13416-401, Piracicaba, SP. Fone: (64) 9641-3617. E-mail: nelson@apagri.com.br

${ }^{2}$ In Memorian

${ }^{3}$ Embrapa Solos, Rua Jardim Botanico 1024, CEP 22460-000, Rio de Janeiro, RJ. E-mail: vinicius.benites@embrapa.br

${ }^{4}$ Instituto Federal Goiano, Campus Iporá, Rodovia GO 060, Km 01, Zona Rural, CEP 76200-000, Iporá, GO. Fone/Fax: (64) 3674-0400. E-mail: relassis@bol.com.br. Bolsista de Produtividade em Pesquisa do CNPq

${ }^{5}$ FESURV, Fazenda Fontes do Saber, CP 104, CEP 75901-970, Rio Verde, GO. Fone: (64) 3611-2262. E-mail: simon@fesurv.br
} 


\section{INTRODUÇÃO}

O sucesso da recomendação de corretivos e fertilizantes depende sobremaneira dos procedimentos adotados na coleta, dos equipamentos utilizados e do preparo da amostra de terra. Portanto, as amostras de solo devem representar adequadamente a área que será corrigida e/ou adubada, para se obter aumento de produtividade e racionalização do uso dos corretivos e fertilizantes (Oliveira et al., 2007).

O revolvimento do solo apenas na linha de plantio, aliado à ação residual da adubação realizada no sulco de semeadura (Alvarez \& Guarçoni, 2003), ao acúmulo da palhada na superfície do solo oriunda da safra de verão quanto da safrinha, à aplicação de adubos e de corretivos na superfície do solo, resulta na formação de gradiente de fertilidade (Schlindwein $\&$ Anghinoni, 2002). Isto proporciona um aumento da variabilidade dos índices de fertilidade (Weirich Neto et al., 2006). Os atributos químicos do solo que apresentam maiores magnitudes de variabilidade do índice de fertilidade são o fósforo, pela menor mobilidade, e o potássio, pela maior movimentação, sendo que esta magnitude vai depender do efeito residual dos corretivos e fertilizantes aplicados, do tipo, da frequência e da quantidade aplicada. Desta forma, grandes diferenças nos valores de teores desses nutrientes entre os locais fertilizados e não-fertilizados, resultam em restrições à aleatorização das amostras simples. A matéria orgânica e o pH em água apresentam menor variabilidade (Schlindwein \& Anghinoni, 2000a).

A estimativa de atributos do solo por meio de avaliações de laboratório depende de técnicas e procedimentos corretos de amostragem. Levando em consideração a maior heterogeneidade devida ao manejo do solo sob o sistema plantio direto, torna-se necessário que se definam métodos de amostragem representativos quanto ao local de coleta, ao volume de terra amostrado, à forma de coleta e ao número de subamostras necessárias para diminuir a variabilidade (Oliveira et al., 2007).

No sistema convencional de cultivo com aração e gradagem o revolvimento constante do solo provoca uma homogeneização na camada mobilizada e menor variabilidade nos gradientes de fertilidade do solo (Schlindwein \& Anghinoni, 2000a).

Os equipamentos mais utilizados na amostragem de solo, no Brasil, são o trado de rosca, o trado holandês, o trado calador (sonda) e a pá-de-corte em que os três primeiros coletam menor volume de solo e são mais práticos no manuseio (Schlindwein \& Anghinoni, 2002). A furadeira elétrica tem sido utilizada com o uso de uma haste de rosca; entretanto, tem-se observado que a quantidade de solo coletada é menor que a de outros equipamentos (Rosolem et al., 2010). Assim e dependendo das análises químicas, alguns tipos de trado podem não apenas ser inadequados mas também necessitar de um aumento no número de subamostras para formar uma amostra composta. No processo de coleta de amostra de solo a quantidade amostrada com o trado holandês, com o de caneca e com a pá-de-corte, é superior a 100 gramas, considerada suficiente para o processo de amostragem.

Segundo Salet et al. (2005) o trado de rosca perde mais solo da camada superficial, com maior coeficiente de variação tornando-se necessário, consequentemente, maior número de subamostras enquanto o trado holandês coleta o dobro de solo da camada superficial, que tem menor coeficiente de variação e é mais prático que a pá-de-corte.

Assim, este trabalho teve por objetivo estudar as variações ocorridas nos resultados de análises químicas de solos cultivados em diferentes locais e períodos de adoção do sistema plantio direto na região de Rio Verde, GO, utilizando-se, para isto, diferentes métodos de amostragem de solo.

\section{Material e Métodos}

Para a realização da pesquisa foram selecionadas 12 áreas em diferentes locais na região de Rio Verde, GO, onde predominam os Latossolos Vermelhos e Latossolos VermelhoAmarelos (EMBRAPA, 1999). As áreas em estudo apresentam relevo plano, com declividade média de $1 \%$ apresentando textura argilosa a muito argilosa.

Em cada uma das áreas selecionadas foram demarcados talhões, com área de um hectare, dentro dos quais foram realizadas aleatoriamente as amostragens de solo, em que cada uma das amostras foi composta de 15 amostras simples.

Os critérios utilizados na escolha das áreas foram: períodos de adoção no sistema plantio direto e disponibilidade para fazer a amostragem de solo após a colheita da soja. Os períodos considerados na seleção das áreas foram 2, 5 e 10 anos de cultivo sob o sistema plantio direto, tendo-se escolhido 4 áreas para cada um dos tempos de cultivo.

Nas áreas selecionadas para a amostragem de solo não foi realizado o plantio da safrinha em 2006, mas foi possível identificar as linhas remanescentes da cultura da soja cultivada no verão, com espaçamento entre linhas de $50 \mathrm{~cm}$, o que serviu de referência para a localização das posições de amostragem.

Os tratamentos consistiram de um fatorial, combinando-se: 2 equipamentos de amostragem (trado holandês e furadeira elétrica), 3 posições (linha de plantio, $1 / 2$ da distância entre as linhas e $1 / 4$ da distância entre as linhas de plantio) e 2 profundidades ( 0 a $10 \mathrm{~cm}$ e 0 a $20 \mathrm{~cm}$ ) de coleta, com 12 repetições para cada tratamento (12 áreas diferentes) totalizando 144 amostras de solo.

As 15 amostras simples de cada tratamento foram colocadas em um recipiente limpo, homogeneizadas e retirados aproximadamente $250 \mathrm{~cm}^{3}$ de cada amostra composta, que foram acondicionadas em sacos plásticos e enviadas ao laboratório, para análise.

Antes de serem analisadas as amostras de solo foram secadas ao ar, destorroadas, passadas em peneira com diâmetro de $2 \mathrm{~mm}$ e homogeneizadas. No laboratório foram determinados o $\mathrm{pH}$, os teores trocáveis de $\mathrm{K}, \mathrm{Ca}, \mathrm{Mg}, \mathrm{Al}$ e $\mathrm{H}+\mathrm{Al}$ e os disponíveis de $\mathrm{P}$; os teores disponíveis de $\mathrm{Fe}, \mathrm{Cu}, \mathrm{Mn}, \mathrm{Zn}$ e B; cálculo da saturação por bases (V), saturação por $\mathrm{Al}(\mathrm{m})$ e capacidade de troca de cátions $(\mathrm{T})$ cujas metodologias são descritas em Silva et al. (1998).

Com o auxílio do programa SISVAR (Ferreira, 2000) os resultados de análises de solo em função dos equipamentos de coleta, profundidades e posições de amostragem foram submetidos à análise de variância e teste de comparação de médias pelo teste de Tukey a 0,05 de probabilidade. 


\section{Resultados E Discussão}

As diferentes formas de amostragem de solo influíram nos resultados analíticos de fertilidade do solo (Tabela 1) o que pode resultar em alterações na interpretação dos laudos emitidos pelos laboratórios de análises de solo.

Os equipamentos trado e furadeira elétrica proporcionaram resultados distintos para todos os macronutrientes, com exceção do $\mathrm{S}$. Os resultados de $\mathrm{pH}, \mathrm{Ca}, \mathrm{Mg}, \mathrm{K}, \mathrm{Al}, \mathrm{H}+\mathrm{Al}, \mathrm{CTC}$, SB, $\mathrm{P}$ e micronutrientes apresentaram diferenças em função do equipamento utilizado. Essas diferenças podem ser atribuídas ao volume de solo coletado por cada equipamento (Silva et al., 2003) especialmente em áreas cultivadas sob o sistema plantio direto onde ocorre uma distribuição irregular de alguns nutrientes, como o $\mathrm{P}$ e $\mathrm{K}$, quando estes são colocados na linha de plantio.

A profundidade de coleta do solo alterou significativamente os valores de $\mathrm{Ca}, \mathrm{Mg}, \mathrm{K}, \mathrm{H}+\mathrm{Al}, \mathrm{P}, \mathrm{MO}, \mathrm{pH}, \mathrm{V}, \mathrm{CTC}, \mathrm{Mn}, \mathrm{Zn}$ e B. A posição de coleta das amostras causou modificação nos valores de $\mathrm{K}, \mathrm{H}+\mathrm{Al} \mathrm{CTC}$, P, S e Fe (Tabela 1).

A aplicação localizada de fertilizantes no sulco de plantio cria duas populações distintas quanto aos teores de nutrientes, sendo uma com elevada concentração e outra com concentração menor, especialmente de nutrientes com pouca mobilidade no solo. O acúmulo de matéria orgânica sobre o solo no sistema plantio direto condiciona a um gradiente de fertilidade nas camadas superficiais. A formação de palha com a utilização de gramíneas e leguminosas com os restos de cultura não incorporados associada à aplicação de corretivos e fertilizantes na superfície, proporciona a presença de camadas de solo com variação vertical de concentração de nutrientes, que diminui com a profundidade (Alvarez \& Guarçoni, 2003). As amostras coletadas na camada de $0-10$ e $0-20 \mathrm{~cm}$ (Tabela 1 ) confirmam a existência de gradiente entre as camadas e corroboram com os dados de literatura.

$\mathrm{O} P$ foi o nutriente que apresentou o gradiente de concentração mais acentuado, tanto horizontal quanto vertical, em função de sua reduzida mobilidade, não sendo transportado no perfil do solo (Silva, 2002). Do ponto de vista de sistema plantio direto, a deposição dos fertilizantes nas camadas superficiais e as menores perdas por erosão (Eltz et al., 1989) justificam o resultado encontrado. Este resultado pode estar associado à aplicação localizada de fertilizantes fosfatados, predominantemente nas linhas de semeadura, que se mantêm pouco alteradas com o tempo de cultivo, devido ao não revolvimento do solo e pela baixa mobilidade do P (Leite et al., 2010).

Além disto, a deposição constante de material orgânico na superfície do solo (Schlindwein \& Anghinoni, 2000b) e a menor fixação do P devido ao reduzido contato deste elemento com os constituintes inorgânicos do solo (Santos \& Tomm, 1996) justificam os teores mais elevados de $P$ obtidos na camada superficial, o que poderia superestimar os teores de $\mathrm{P}$ se a amostragem de solo fosse realizada a apenas até $10 \mathrm{~cm}$ de profundidade.

Apesar das constantes operações de plantio e da formação de linhas de concentração de fertilizantes em posições diferentes, não ocorreu homogeneização dos teores de $\mathrm{P}$ na superfície do terreno ao longo dos anos de adoção do sistema plantio direto (Tabela 2).

As variações na distribuição deste mineral, tanto horizontal como vertical, ocorreram em todos os períodos, constatandose maior concentração na linha de plantio e na camada até 10 $\mathrm{cm}$ de profundidade, independente do período de adoção do sistema plantio direto.

Além do $\mathrm{P}$ alguns nutrientes de baixa mobilidade no solo, como Ca e Mg (Lima et al., 2013) também podem acumular-

Tabela 1. Média dos resultados das análises químicas do solo $(n=144)$ em função do equipamento, profundidade e posição utilizados para a coleta das amostras de solo

\begin{tabular}{|c|c|c|c|c|c|c|c|}
\hline \multirow[b]{2}{*}{ Variável } & \multicolumn{7}{|c|}{ Tratamentos } \\
\hline & \multicolumn{2}{|c|}{ Equipamento } & \multicolumn{2}{|c|}{ Profundidade (cm) } & \multicolumn{3}{|c|}{ Posição } \\
\hline $\mathrm{pH}$ & $5,13 b$ & $5,23 \mathrm{a}$ & $5,20 \mathrm{a}$ & $5,15 b$ & $5,20 \mathrm{a}$ & $5,18 \mathrm{a}$ & $5,15 \mathrm{a}$ \\
\hline $\mathrm{Ca}$ & $2,73 b$ & $3,13 \mathrm{a}$ & $3,05 \mathrm{a}$ & $2,81 \mathrm{~b}$ & $2,92 \mathrm{a}$ & $2,92 \mathrm{a}$ & $2,95 \mathrm{a}$ \\
\hline $\mathrm{Mg}$ & $0,95 b$ & $1,18 \mathrm{a}$ & $1,11 \mathrm{a}$ & $1,02 b$ & $1,02 \mathrm{a}$ & $1,08 \mathrm{a}$ & $1,09 \mathrm{a}$ \\
\hline K & $0,18 b$ & $0,25 \mathrm{a}$ & $0,23 \mathrm{a}$ & $0,20 \mathrm{~b}$ & $0,19 \mathrm{~b}$ & $0,20 \mathrm{~b}$ & $0,27 \mathrm{a}$ \\
\hline CTC & $7,57 \mathrm{~b}$ & $8,05 \mathrm{a}$ & $7,92 \mathrm{a}$ & $7,70 \mathrm{~b}$ & $7,72 \mathrm{~b}$ & $7,79 a b$ & $7,92 \mathrm{a}$ \\
\hline $\mathrm{SB}$ & $3,86 \mathrm{~b}$ & $4,56 \mathrm{a}$ & $4,39 \mathrm{a}$ & $4,03 \mathrm{~b}$ & $4,20 \mathrm{a}$ & $4,21 \mathrm{a}$ & $4,22 \mathrm{a}$ \\
\hline & & & & $\mathrm{mg} \mathrm{dm}^{-3}$ & & & \\
\hline$P$ & $10,73 b$ & $13,68 \mathrm{a}$ & $14,38 \mathrm{a}$ & $10,03 b$ & $8,34 \mathrm{~b}$ & $8,70 \mathrm{~b}$ & $19,58 \mathrm{a}$ \\
\hline$S$ & $7,97 \mathrm{a}$ & $8,59 \mathrm{a}$ & $7,83 \mathrm{a}$ & $8,72 \mathrm{a}$ & $7,86 a b$ & $7,14 \mathrm{~b}$ & $9,83 \mathrm{a}$ \\
\hline $\mathrm{Fe}$ & $37,00 \mathrm{~b}$ & $38,81 \mathrm{a}$ & $37,67 \mathrm{a}$ & $38,14 \mathrm{a}$ & $36,51 \mathrm{~b}$ & $37,12 \mathrm{~b}$ & $40,09 \mathrm{a}$ \\
\hline MO & $37,30 \mathrm{~b}$ & $40,89 a$ & $40,04 \mathrm{a}$ & $\begin{array}{r}\mathrm{g} \mathrm{dm}^{-3} \\
38,15 \mathrm{~b}\end{array}$ & $39,76 \mathrm{a}$ & $38,73 \mathrm{a}$ & $38,80 \mathrm{a}$ \\
\hline V & $51,19 \mathrm{~b}$ & $56,46 \mathrm{a}$ & $55,51 \mathrm{a}$ & $\begin{array}{c}\% \\
52,14 b \\
\end{array}$ & $54,37 \mathrm{a}$ & $53,93 \mathrm{a}$ & $53,17 \mathrm{a}$ \\
\hline
\end{tabular}

Médias seguidas pela mesma letra não diferem entre si (Tukey a 0,05$)$ dentro da mesma fonte de variação 
Tabela 2. Atributos químicos do solo em função do equipamento, profundidade, posição e período de adoção do sistema plantio direto

\begin{tabular}{|c|c|c|c|c|c|c|c|}
\hline \multirow{3}{*}{ Variável } & \multicolumn{7}{|c|}{ Tratamentos } \\
\hline & \multicolumn{2}{|c|}{ Equipamento } & \multicolumn{2}{|c|}{ Profundidade (cm) } & \multicolumn{3}{|c|}{ Posição } \\
\hline & Trado & Furadeira & $0-10$ & $0-20$ & $1 / 2$ dist. linha & 1/4 dist. linha & Linha \\
\hline & & & & $\begin{array}{l}\operatorname{nos}(\mathrm{n}= \\
\mathrm{cmol} \mathrm{d}_{\mathrm{c}} \mathrm{dm}\end{array}$ & & & \\
\hline $\mathrm{Ca}$ & $2,99 \mathrm{~b}$ & $3,28 \mathrm{a}$ & $3,27 \mathrm{a}$ & $3,01 \mathrm{~b}$ & $3,06 \mathrm{a}$ & $3,08 \mathrm{a}$ & $3,27 \mathrm{a}$ \\
\hline $\mathrm{Mg}$ & $1,04 b$ & $1,23 \mathrm{a}$ & $1,16 \mathrm{a}$ & $1,10 \mathrm{a}$ & $1,13 \mathrm{a}$ & $1,16 \mathrm{a}$ & $1,11 \mathrm{a}$ \\
\hline $\mathrm{K}$ & $0,21 \mathrm{a}$ & $0,23 \mathrm{a}$ & $0,23 \mathrm{a}$ & $0,20 \mathrm{~b}$ & $0,19 \mathrm{~b}$ & $0,21 \mathrm{~b}$ & $0,25 \mathrm{a}$ \\
\hline $\mathrm{P}$ & $9,79 \mathrm{~b}$ & $11,42 \mathrm{a}$ & $12,72 \mathrm{a}$ & $\begin{array}{c}\mathrm{mg} \mathrm{dm}^{-3} \\
8,49 \mathrm{~b}\end{array}$ & $7,24 \mathrm{~b}$ & $6,91 \mathrm{~b}$ & $17,67 \mathrm{a}$ \\
\hline MO & $36,36 \mathrm{a}$ & $37,40 \mathrm{a}$ & $37,62 \mathrm{a}$ & $\begin{array}{r}\mathrm{g} \mathrm{dm}^{-3} \\
36,15 \mathrm{a}\end{array}$ & $37,22 \mathrm{a}$ & $36,06 \mathrm{a}$ & $37,38 \mathrm{a}$ \\
\hline & & & & $\begin{array}{l}\text { nos }(\mathrm{n}= \\
\mathrm{cmol} \mathrm{dm}_{\mathrm{c}}\end{array}$ & & & \\
\hline $\mathrm{Ca}$ & $2,41 \mathrm{~b}$ & $2,61 \mathrm{a}$ & $2,59 \mathrm{a}$ & $2,43 \mathrm{~b}$ & $2,51 \mathrm{a}$ & $2,56 \mathrm{a}$ & $2,46 \mathrm{a}$ \\
\hline $\mathrm{Mg}$ & $0,80 \mathrm{~b}$ & $0,94 \mathrm{a}$ & $0,91 \mathrm{a}$ & $0,83 \mathrm{a}$ & $0,89 a$ & $0,91 \mathrm{a}$ & $0,81 \mathrm{a}$ \\
\hline $\mathrm{K}$ & $0,13 b$ & $0,20 \mathrm{a}$ & $0,18 \mathrm{a}$ & $0,15 \mathrm{~b}$ & $0,16 \mathrm{a}$ & $0,16 \mathrm{a}$ & $0,19 a$ \\
\hline $\mathrm{P}$ & $10,50 \mathrm{a}$ & $10,88 \mathrm{a}$ & $12,25 \mathrm{a}$ & $\begin{array}{c}\mathrm{mg} \mathrm{dm}^{-3} \\
9,14 \mathrm{~b}\end{array}$ & $6,34 \mathrm{~b}$ & $7,43 \mathrm{~b}$ & $18,31 \mathrm{a}$ \\
\hline MO & $29,06 \mathrm{~b}$ & $32,87 \mathrm{a}$ & $31,93 \mathrm{a}$ & $\begin{array}{r}\mathrm{g} \mathrm{dm}^{-3} \\
30,02 \mathrm{~b}\end{array}$ & $30,99 \mathrm{a}$ & $31,22 \mathrm{a}$ & $30,71 \mathrm{a}$ \\
\hline & & & & $\begin{array}{l}\text { anos }(\mathrm{n}= \\
\mathrm{cmol}_{\mathrm{c}} \mathrm{dm}\end{array}$ & & & \\
\hline $\mathrm{Ca}$ & $2,79 \mathrm{~b}$ & $3,49 a$ & $3,29 a$ & $3,00 \mathrm{~b}$ & $3,19 a$ & $3,14 \mathrm{a}$ & $3,11 \mathrm{a}$ \\
\hline $\mathrm{Mg}$ & $1,01 b$ & $1,35 \mathrm{a}$ & $1,24 \mathrm{a}$ & $1,13 \mathrm{~b}$ & 1,23 a & $1,17 \mathrm{a}$ & $1,15 \mathrm{a}$ \\
\hline $\mathrm{K}$ & $0,20 \mathrm{~b}$ & $0,33 \mathrm{a}$ & $0,29 \mathrm{a}$ & $0,25 \mathrm{~b}$ & $0,22 \mathrm{~b}$ & $0,26 \mathrm{~b}$ & $0,33 \mathrm{a}$ \\
\hline $\mathrm{P}$ & $11,90 \mathrm{~b}$ & $18,75 \mathrm{a}$ & $18,18 \mathrm{a}$ & $\begin{array}{l}\mathrm{mg} \mathrm{dm}^{-3} \\
12,47 \mathrm{~b}\end{array}$ & $11,45 \mathrm{~b}$ & $11,75 b$ & $22,77 \mathrm{a}$ \\
\hline MO & $46,47 \mathrm{~b}$ & $52,39 a$ & $50,58 \mathrm{a}$ & $\begin{array}{c}\mathrm{g} \mathrm{dm}^{-3} \\
48,28 \mathrm{~b}\end{array}$ & $50,90 \mathrm{a}$ & $48,91 \mathrm{ab}$ & $48,47 \mathrm{~b}$ \\
\hline
\end{tabular}

Médias seguidas pela mesma letra na linha não diferem entre si (Tukey a 0,05) dentro da mesma fonte de variação

se na superfície criando um gradiente de concentração que interfere no resultado de análise de solo para fins de fertilidade (Amaral \& Anghinoni, 2001).

Os resultados mais elevados de $\mathrm{Ca}, \mathrm{Mg}$ e $\mathrm{pH}$ na profundidade de 0 a $10 \mathrm{~cm}$ são consequência da aplicação superficial de calcário, sem posterior incorporação (Silveira \& Stone, 2002). Mesmo nas áreas cultivadas há 10 anos sob o sistema plantio direto, os teores de $\mathrm{Ca}$ e $\mathrm{Mg}$ são mais elevados nas camadas superficiais em função das calagens superficiais e reduzida movimentação do calcário no perfil do solo.

As calagens também podem ter contribuído para os teores mais elevados de $\mathrm{K}$ na superfície, uma vez que o uso do calcário gera cargas negativas e, em contrapartida, aumenta a retenção de cátions (Souza et al., 2007).

Os teores mais elevados de $\mathrm{K}$ na linha de plantio e na superfície se mantêm nas áreas com 10 anos de cultivo sob o sistema plantio direto (Tabela 2) o que pode ser atribuído à distribuição localizada do adubo na linha de plantio. A localização das plantas também contribuiu para sua variabilidade uma vez que este elemento não forma compostos orgânicos no tecido sendo então facilmente transportado da parte aérea para o solo, pela chuva no final do ciclo da planta, concentrando o K próximo ao colo da mesma (Klepker \& Anghinoni, 1993). Entretanto, além do K se diluir parcialmente na água e sendo transportado para camadas mais profundas, a maior quantidade se encontra na camada superficial, a até $5 \mathrm{~cm}$ de profundidade (Anghinoni, 2007).

Os valores de MOS nos primeiros $10 \mathrm{~cm}$ do perfil do solo também são mais elevados quando comparados aos obtidos na amostragem realizada até $20 \mathrm{~cm}$ de profundidade. A deposição dos restos vegetais deve ter contribuído para o enriquecimento desta camada do solo uma vez que a manutenção do material orgânico na superfície do terreno também é responsável por grande parte da maior disponibilidade de nutrientes nos primeiros $5 \mathrm{~cm}$ do perfil do solo.

No sistema plantio direto o acúmulo de resíduos de culturas em superfície, sem o revolvimento do solo, altera a taxa de decomposição da matéria orgânica e a liberação de nutrientes aumentando consideravelmente os teores de carbono. Aliado às frequentes adubações nas linhas de cultivo e calagens em superfície forma um gradiente de concentração superficial de matéria orgânica e nutrientes aumentando tanto a variabilidade vertical quanto a horizontal (Zanão Júnior et al., 2010).

Portanto, a distribuição superficial do calcário e da palhada no sistema plantio direto induz a uma baixa variabilidade nos atributos de acidez e do teor de MO, o que se reflete em resultados semelhantes para as variáveis $\mathrm{Ca}, \mathrm{Mg}$ e $\mathrm{MO}$, independente da posição de amostragem do solo (Salet et al., 2005).

Além das variações nos resultados analíticos em função da posição na superfície e da profundidade de coleta, os equipamentos utilizados na amostragem podem ser fonte de variabilidade dos resultados de análise de solo, sobretudo para os elementos $\mathrm{P}$ e $\mathrm{K}$ que apresentam alta variabilidade, independente do equipamento utilizado para realizar a amostragem do solo (Alvarez \& Guarçoni, 2003).

A existência de interação significativa entre profundidade e equipamento demonstra que as ferramentas utilizadas neste 
Tabela 3. Atributos químicos do solo, influenciados pela interação entre equipamento e profundidade usados na amostragem de solo

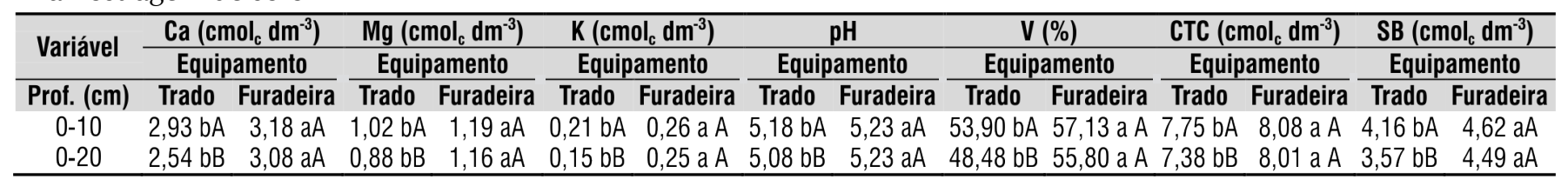

Médias seguidas pela mesma letra, maiúscula na coluna e minúscula na linha, não diferem pelo teste de Tukey a 0,05 de probabilidade

trabalho para a coleta de solo apresentaram desempenho diferenciado constituindo-se, portanto, fonte de variabilidade (Tabela 3).

Por outro lado, o trado foi mais sensível ao gradiente de fertilidade em que, normalmente, se forma em áreas sob plantio direto (Klepker \& Anghinoni, 1993; Eltz et al., 1989; Amaral \& Anghinoni, 2001) provocado pelas calagens superficiais que alteram o nível de diversas variáveis químicas do solo (Souza et al., 2007) e pelas adubações superficiais que contribuem para a formação de um gradiente de Pe K (Schlindwein \& Anghinoni, 2000b) promovendo variabilidade de $\mathrm{pH}$ e dos teores de vários minerais (Eltz et al., 1989).

Como no sistema plantio direto há maior disponibilidade de nutrientes nos primeiros $5 \mathrm{~cm}$ do perfil do solo, as amostras retiradas com a furadeira representaram apenas os índices de fertilidade da camada em que há maior concentração de nutrientes mudando, inclusive, a faixa de interpretação do K e $\mathrm{P}$, resultando em recomendações errôneas influenciando, então, na produtividade e rentabilidade das culturas.

Os teores do $\mathrm{P}$ foram influenciados significativamente pela interação entre posição e profundidade de amostragem, sendo mais elevados na linha de plantio que os obtidos nas outras posições, tal como as amostras retiradas na linha e a $1 / 4$ da distância da linha apresentaram valores mais elevados na camada superficial (Tabela 4). Em linha, a adubação fosfatada cria dois padrões de concentração de fósforo, um na linha e o outro na entrelinha; então, a amostragem deve ser realizada de modo a ser representativa (Anghinoni, 2007).

A baixa mobilidade do $\mathrm{P}$ e a manutenção das linhas de adubação dos plantios anteriores decorrentes do nãorevolvimento do solo exerceram grande influência na interação significativa entre as variáveis posição e profundidade (Tabela 4).

Tabela 4. Teores de P pelo teste de Tukey em função da profundidade de coleta e da posição de amostragem

\begin{tabular}{cccc}
\hline \multirow{2}{*}{ Tratamento } & \multicolumn{3}{c}{$\mathbf{P}\left(\mathbf{m g ~ d m}^{-3}\right)$} \\
\cline { 2 - 4 } & \multicolumn{3}{c}{ Posição } \\
\hline Prof. (cm) & Linha & $\mathbf{1 / 4}$ linha & $1 / 2$ linha \\
$0-10$ & $23,42 \mathrm{aA}$ & $10,45 \mathrm{bA}$ & $9,28 \mathrm{bA}$ \\
$0-20$ & $15,75 \mathrm{aB}$ & $6,94 \mathrm{bB}$ & $7,41 \mathrm{bA}$ \\
\hline
\end{tabular}

Médias seguidas pela mesma letra, maiúscula na coluna e minúscula na linha, não diferem pelo teste de Tukey a 0,05 de probabilidade

As amostragens realizadas exclusivamente na linha de plantio ou fora dela, como as deste trabalho, parecem não representar adequadamente a fertilidade do solo sob o sistema plantio direto. Quando as amostras abrangem uma superfície maior, inclusive a linha de plantio, os valores dos índices de fertilidade também são maiores sendo que as diferenças são mais acentuadas para os elementos P e K (Salet et al., 2005).
A amostragem realizada com equipamentos que coletam um volume reduzido de solo exige uma combinação de posições, conforme observado por Alvarez \& Guarçoni (2003). Esses autores obtiveram índice de fertilidade semelhante ao da páde-corte quando a amostragem realizada com o trado de caneca foi obtida com $17 \%$ das amostras simples coletadas no sulco de plantio, $33 \%$ a $10 \mathrm{~cm}$ do sulco e $50 \%$ no ponto médio entre os sulcos.

Portanto, a posição, a profundidade e o equipamento de coleta de solo podem mudar substancialmente os resultados de análise de solo e em consequência as recomendações de adubação.

A maior variabilidade desses índices de fertilidade significa maior número de amostras simples para formar uma amostra composta. Portanto, esta etapa é crítica na utilização das recomendações de adubação e calagem haja vista que os erros contidos na amostra não mais poderão ser corrigidos resultando em recomendações de quantidades insuficientes ou excessivas de insumos, que se refletirão em qualquer um dos casos, em prejuízos no rendimento das culturas e/ou no lucro do produtor (Anghinoni, 2007).

\section{Conclusões}

1. O sistema plantio direto provocou um gradiente superficial de fertilidade para todos os macronutrientes, com exceção do $\mathrm{S}$, em função das calagens, deposição de palhada e adubações superficiais.

2. As adubações na linha de plantio também provocaram um gradiente horizontal de fertilidade acentuado, em especial para os elementos $\mathrm{P}$ e $\mathrm{K}$.

3. As áreas cultivadas há 10 anos sob o sistema plantio direto apresentaram um gradiente de fertilidade no perfil demonstrando a reduzida mobilidade dos elementos $\mathrm{Ca}, \mathrm{Mg}$, $\mathrm{K}$ e $\mathrm{P}$ aplicados na superfície do solo.

4. Os equipamentos utilizados na amostragem influíram nos resultados de análise de solo sendo que o trado representou melhor o gradiente de fertilidade que se formou com a adoção do sistema plantio direto.

5. As sucessivas operações de plantio ao longo dos anos não causaram uma homogeneização horizontal dos elementos $\mathrm{K}$ e $\mathrm{P}$ aplicados na linha de plantio.

\section{Agradecimentos}

À Fundação AGRISUS, pelo auxílio financeiro na forma de bolsa de estudo, concedido ao primeiro autor deste trabalho de pesquisa. 


\section{Literatura Citada}

Alvarez V. H.; Guarçoni, M. Variabilidade horizontal da fertilidade do solo de uma unidade de amostragem em sistema de plantio direto. Revista Brasileira de Ciência do Solo, v.27, p.297-310, 2003.

Amaral, A. S.; Anghinoni, I. Alterações de parâmetros químicos do solo pela reaplicação superficial de calcário no sistema plantio direto. Pesquisa Agropecuária Brasileira, v.36, p.695-702, 2001.

Anghinoni, I. Fertilidade do solo e seu manejo em sistema plantio direto. In: Novais, R. F.; Alvarez V., V. H.; Barros, N. F.; Fontes, R. L. F.; Cantarutti R. B.; Neves, J. C. L., eds. Fertilidade do solo. Viçosa: Sociedade Brasileira de Ciência do Solo, 2007. p.873-928.

Eltz, F. L. P.; Peixoto, R. T. G.; Jaster, F. Efeitos de sistemas de preparo do solo nas propriedades físicas e químicas de um Latossolo Bruno álico. Revista Brasileira de Ciência do Solo, v.13, p.259-267, 1989.

EMBRAPA - Empresa Brasileira de Pesquisa Agropecuária. Centro Nacional de Pesquisa de Solos (Rio de Janeiro, RJ). Sistema brasileiro de classificação de solos. Brasília: Embrapa Produção de Informação, 1999. 412p.

Ferreira, D. F. Análises estatísticas por meio do Sisvar para Windows versão 4.0. In: Reunião Anual da Região Brasileira da Sociedade internacional de Biometria, 45, 2000, São Carlos. Anais... São Carlos: UFSCar, 2000. p.255-258.

Klepker, D.; Anghinoni, I. Phosphate uptake and corn root distribution as affected by fertilizer placement and soil tillage. Agronomy Trends Agricultural Science, v.1, p.111$115,1993$.

Leite, L. F. C.; Galvão, S. R. S.; Holanda Neto, M. R.; Araújo, F. S.; Iwata, B. F. Atributos químicos e estoques de carbono em Latossolo sob plantio direto no cerrado do Piauí. Revista Brasileira de Engenharia Agrícola e Ambiental, v.14, p.1273-1280, 2010

Lima, J. S. de S.; Silva, S. de A.; Silva, J. M. da. Variabilidade espacial de atributos químicos de um Latossolo VermelhoAmarelo cultivado em plantio direto. Revista Ciência Agronômica, v.44, p.16-23, 2013.

Oliveira, F. H. T.; Arruda, J. A. de; Silva, I. F. da; Alves, J. C. Amostragem para avaliação da fertilidade do solo em função do instrumento de coleta das amostras e de tipos de preparo do solo. Revista Brasileira de Ciência do Solo, v.31, p.973-983, 2007.

Rosolem, C. A.; Tozi, T. de S.; Garcia, R. A. Amostragem de terra para fins de fertilidade em função da ferramenta de amostragem. Revista Ceres, v.57, p.405-414, 2010.
Salet, R. L.; Nicolodi, M.; Bisso, F. P. Eficácia do trado holandês na amostragem de solo em lavouras no sistema plantio direto. Revista Brasileira de Agrociência, v.11, p.487-491, 2005.

Santos, H. P.; Tomm, G. O. Estudo da fertilidade do solo sob quatro sistemas de rotação de culturas envolvendo trigo em plantio direto. Revista Brasileira de Ciência do Solo, v.20, p.407-414, 1996.

Schlindwein, J. A.; Anghinoni, I. Variabilidade horizontal de atributos de fertilidade e amostragem do solo no sistema plantio direto. Revista Brasileira de Ciência do Solo, v.24, p.85-91, 2000a.

Schlindwein, J. A.; Anghinoni, I. Variabilidade vertical do fósforo e potássio disponíveis e profundidade de amostragem do solo no sistema plantio direto. Ciência Rural, v.30, p.611-617, 2000b

Schlindwein, J. A.; Anghinoni, I. Tamanho da subamostra e representatividade da fertilidade do solo no sistema plantio direto. Ciência Rural, v.32, p.963-968, 2002.

Silva, F. C.; Eira, P. A.; Barreto, W. O.; Pérez, D. V.; Silva, C. A. Manual de métodos de análises químicas para avaliação da fertilidade do solo. Rio de Janeiro: Embrapa CNPS, 1998. 56p. Documentos, 3

Silva, M. A. G., Muniz, A. S., Sengik, E., Mata, J. D. V., Carassini, C., Cegana, A.C. Amostragem e variabilidade nos atributos de fertilidade em um Latossolo sob plantio direto em São Miguel do Iguaçu, Estado do Paraná. Acta Scientiarum: Agronomy. v.25, p.243-248, 2003.

Silva, M. L. S. Sistema de amostragem do solo e avaliação da disponibilidade de fósforo na fase de implantação do plantio direto. Piracicaba: ESALQ/USP, 2002. 111p. Dissertação Mestrado

Silveira, P. M.; Stone, L. F. Profundidade de amostragem do solo sob plantio direto para avaliação de características químicas. Santo Antônio de Goiás: EMBRAPA, 2002. 2p. Comunicado técnico 37.

Souza, D. M. G. de; Miranda, L. N. de; Oliveira, S. A. de. Acidez do solo e sua correção. In: Novais, F. N.; Alvarez V., V. H.; Barros, N. F. de; Fontes, R. L. F.; Cantarutti, R. B.; Neves, J. C. L. Fertilidade do solo. Viçosa: Sociedade Brasileira de Ciência do Solo, 2007. p.205-274.

Weirich Neto, P. H.; Sverzut, C. B.; Schimandeiro, A. Necessidade de fertilizante e calcário em área sob sistema plantio direto considerando variabilidade espacial. Revista Brasileira de Engenharia Agrícola e Ambiental, v.10, p.338-343, 2006.

Zanão Júnior, L. A.; Lana, R. M. Q.; Guimarães, E. C.; Araújo, J. M. de. Variabilidade espacial dos teores de macronutrientes em Latossolos sob sistema plantio direto. Revista Brasileira de Ciência do Solo, v.34, p.389-400, 2010. 